\title{
Diabetic Ocular Complications in the SDT Rat
}

\author{
Akihiro Kakehashi* \\ Department of Ophthalmology, Saitama Medical Center, Jichi Medical University, Saitama, Japan
}

\begin{abstract}
The purpose of the current article was to describe the diabetic ocular complications in a new animal model of diabetes, the Spontaneously Diabetic Torii (SDT) rat. Three major diabetic ocular complications, cataract, diabetic retinopathy (DR), and neovascular glaucoma develop in the SDT rat. The cataract develops to maturity, and DR develops to the advanced stage. Large retinal folds mimicking a tractional retinal detachment with extensive fluorescein leakage around the optic disc are the most characteristic finding of DR in this rat. In some rats, neovascular glaucoma develops with a massive hemorrhage in the anterior chamber associated with neovascular fibrous membranes around the iris. Although there are some differences in the diabetic ocular complications between SDT rats and human patients with diabetes, the SDT rat model is useful for studying diabetic ocular complications.
\end{abstract}

Keywords: Cataract, diabetic retinopathy, SDT rat, diabetic ocular complication, animal model of diabetes.

\section{INTRODUCTION}

Numerous ocular complications develop in patients with diabetes, such as cataract, diabetic retinopathy (DR), neovascular glaucoma, optic neuropathy, keratopathy, pupillomotor disturbance, external ophthalmoplegia, accommodation insufficiency, and uveitis; the most frequent of these are cataract, DR, and neovascular glaucoma. Most cataracts can be treated safely by cataract surgery. However, DR is still a leading cause of visual loss and blindness in adults in most developed countries [1]. Neovascular glaucoma, which usually follows the development of severe proliferative diabetic retinopathy (PDR) and is assumed to result from advanced retinal hypoxia, is the most severe diabetic ocular complication, because it is associated with PDR and is difficult to treat even with panretinal photocoagulation, vitreous surgery, glaucoma surgery, or all of these.

Diabetic ocular complications do not develop if glycemic control is achieved at the onset of the disease [2, 3]. However, many patients pay little attention to poor glycemic control until their vision becomes compromised. Therefore, to prevent diabetic ocular complications, prophylactic medical treatments are needed other than glycemic control. To accomplish this, a spontaneously diabetic animal model with severe diabetic complications should be developed, but no such animal model exists. Although numerous spontaneous diabetic animal models have been reported, few models develop ocular complications. Among them, some experimental diabetic dogs and galactose-fed dogs develop DR $[4,5]$. Although these dog models of diabetes have features characteristic of DR, they have not been used widely in experimental studies of DR because of the low prevalence of DR and the prolonged time needed for the complication to develop. Moreover, the dog model is inconvenient and costly

*Address correspondence to this author at the Department of Ophthalmology, Saitama Medical Center, Jichi Medical University, Saitama, 330-8503, Japan; Tel: +81-48-647-2111; Fax: +81-648-5188;

E-mail:kakeaki@omiya.jichi.ac.jp for large-scale experiments. In contrast, rat and mouse models are popular for many types of experimental diabetic studies. However, most diabetic rat or mouse models develop only subclinical DR. The Spontaneously Diabetic Torii (SDT) rat is the first animal model with advanced DR and other diabetic ocular complications [6-8]. The male SDT rats develop marked hyperglycemia (about $700 \mathrm{mg} / \mathrm{dL}$ ) and glucosuria after 20 weeks of age. The cumulative incidence of diabetes is almost $100 \%$ by 40 weeks of age in male SDT rats. The SDT rats will be useful to study the pathogenesis and treatment of DR, although further investigation is needed to clarify the mechanisms of the differences between this model and patients with diabetes.

\section{CATARACT}

Cataract is the most frequently occurring ocular complication in patients with diabetes. Two types of cataract develop, one of which is indistinguishable from senile cataract. The other is a mature cataract. The latter is considered "typical" of diabetes and related to the severity of the disease.

Several types of cataract, i.e., cortical, nuclear, and subcapsular, are observed in SDT rats, after the development of diabetes. The cataracts mature in most SDT rats by the time the animals reach 40 weeks of age (Figs. 1, 2). A pathological study showed that the sclerotic nucleus of the lens floats in a liquefied lens cortex (Fig. 3) when the cataracts mature. Vacuolation, disintegration of the lens fibers, and Morgani's globules were observed in the lens cortex. Some advanced cases of hypermature cataract include rupture of the posterior capsule, which may accelerate the development of preretinal proliferation and subsequent tractional retinal detachment. All of these features make the SDT rat a good animal model of diabetic cataract.

\section{DIABETIC RETINOPATHY}

DR in humans progresses gradually from the early to the advanced stages. The early stage in humans is characterized by development of microaneurysms and retinal hemorrhages 
resulting from microvascular dysfunction. The microaneurysms are thought to be the initial stage of retinal neovascularization. Cotton-wool spots appear as early evidence of retinal ischemia, which represents arrest of the axoplasmic flow in the retinal nerve fiber layer at the margin of a microvascular occlusion. When the capillary nonperfusion extends to the major veins, there is irregularity of the venous caliber in the retinal veins, known as venous beading or venous segmentation. With more extensive capillary nonperfusion, retinal neovascularization, which is characterized by formation of a net- or funnel-like vascular structure, arises from the optic disc (new vessels of the disc [NVD]) and/or other retinal areas (new vessels elsewhere [NVE]). Extensive leakage of blood components from the NVE and NVD induce vitreous shrinkage and a posterior vitreous detachment (PVD). However, because of strong adhesion between the retina and vitreous cortex at the site of NVD and/or NVE, the PVD progresses incompletely and induces a tractional retinal detachment. The vitreous shrinkage and tractional retinal detachment worsen with the recruitment of fibrous elements around the NVD and NVE. Thus, massive vitreous hemorrhages and preretinal hemorrhages from the NVD and NVE are seen frequently in this stage.

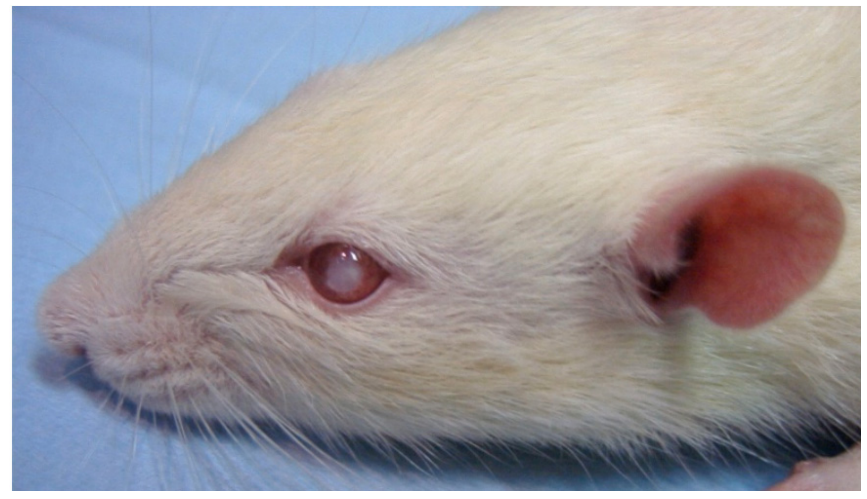

Fig. (1). External appearance of a SDT rat. A mature milky cataract is seen in a SDT rat.

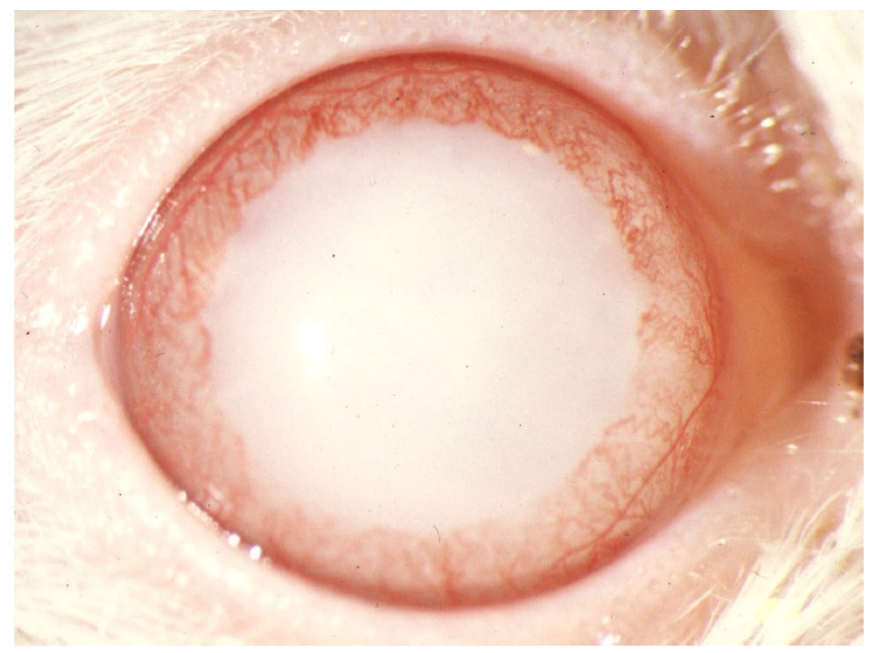

Fig. (2). A mature cataract in a SDT rat. The mature milky cataract is observed clearly in the dilated pupil in a 52-year-old male SDT rat [8].

The DR that develops in SDT rats differs from that in humans. The retinal hemorrhages and microaneurysms do not develop frequently in SDT rats. The retinal vasculature evaluated in trypsin-digested retinal specimens showed pericyte loss and occluded capillaries (Fig. 4) similar to the DR in humans. The venous beading or venous segmentation with extensive dye leakage was seen frequently on fluorescein microangioscopy (Fig. 5) after the animals reached 50 weeks of age. The tractional retinal detachment with large folds of thickened retina was seen frequently around the optic disc (Fig. 6). These findings are the most typical of advanced retinopathy in SDT rats. A tractional retinal detachment was observed in the midperiphery of the retina (Fig. 7) associated with extensive dye leakage (Fig. 8), but its incidence is rare. Capillary occlusion was observed around the optic disc in some SDT rats, but it was not a frequent finding. The early diabetic retinal changes observed in DR in humans, such as microaneurysms, small retinal hemorrhages, and capillary nonperfusion, seem to be bypassed in the SDT rats. The DR that develops in SDT rats, i.e., proliferative DR without marked retinal ischemia, may result from increased expression of both vascular endothelial growth factor (VEGF) and pigment epithelium-derived factor $[9,10]$. The prevalence of advanced retinopathy in SDT rats was about $80 \%$ by age 60 weeks, but it was rarely observed before 50 weeks of age.

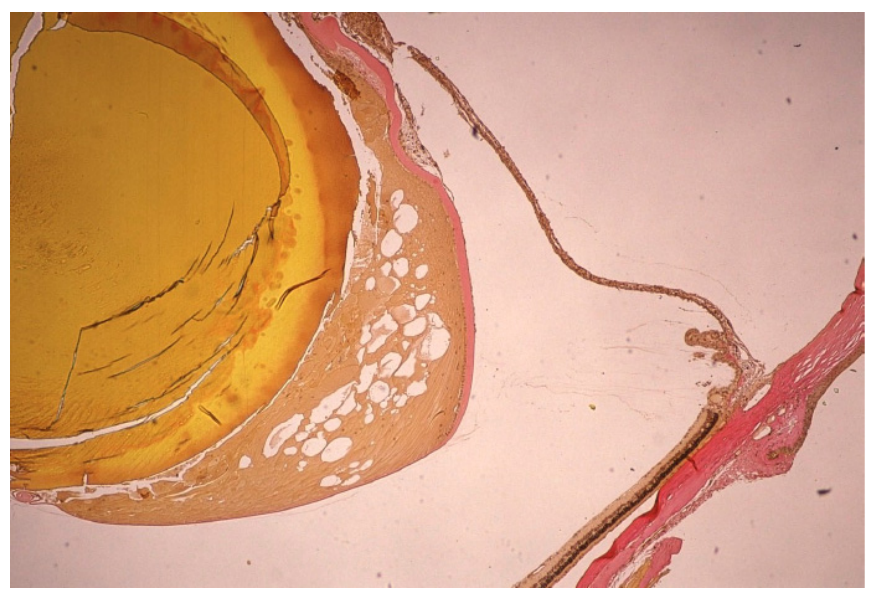

Fig. (3). Pathology of the mature cataract in a SDT rat. The sclerotic nucleus floats in a liquefied lens cortex. Vacuolation, disintegration of the lens fibers, and Morgani's globules are observed in the lens cortex (elastica van Gieson stain, 4x).
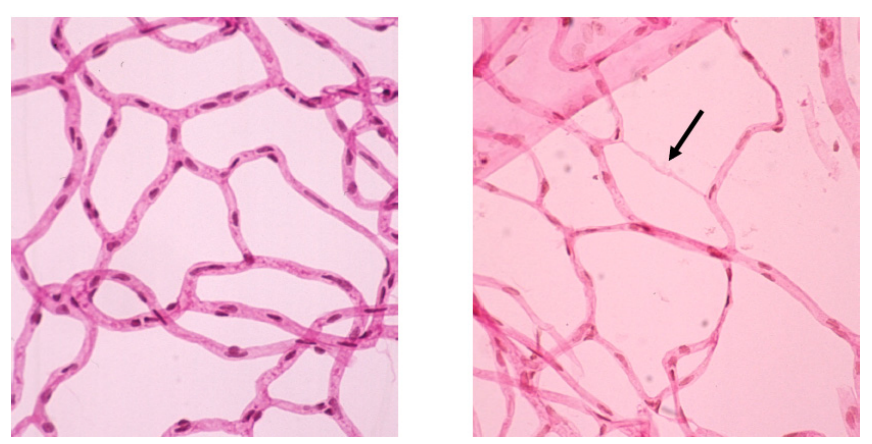

Fig. (4). Trypsin digested retina of the SDT rat. Left: A 36-weekold male SDT rat has normal retinal vasculature. The endothelial cell/pericyte ratio of the retinal capillary is almost one to one. Right: A 51-week-old male SDT rat has pericyte loss and acellular capillaries (arrow). There is extensive narrowing of the capillaries. However, no capillary microaneurysms are seen as is common in human diabetic retinopathy (periodic acid-Schiff stain, 40x) [6]. 

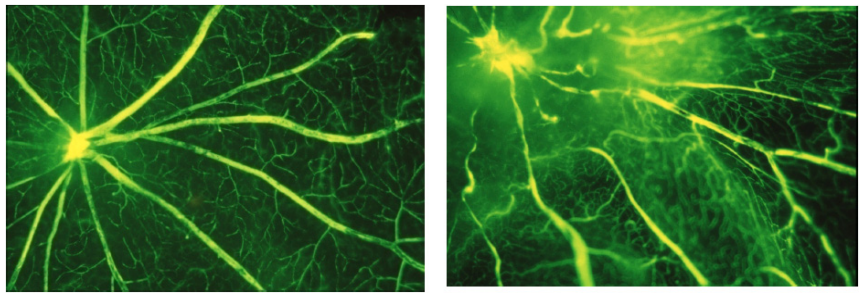

Fig. (5). Fluorescein-dextran angiomicroscopy around the optic disc in a SDT rat. Left: Normal findings on fluorescein-dextran angiomicroscopy are seen in a 36-week-old male SDT rat. Right: Extensive hyperfluorescence is observed in a 51-week-old male SDT rat. Extensive dye leakage from the retinal vessels usually is accompanied by capillary occlusion in humans, but angiographic capillary occlusion is not as evident in SDT rats [6].

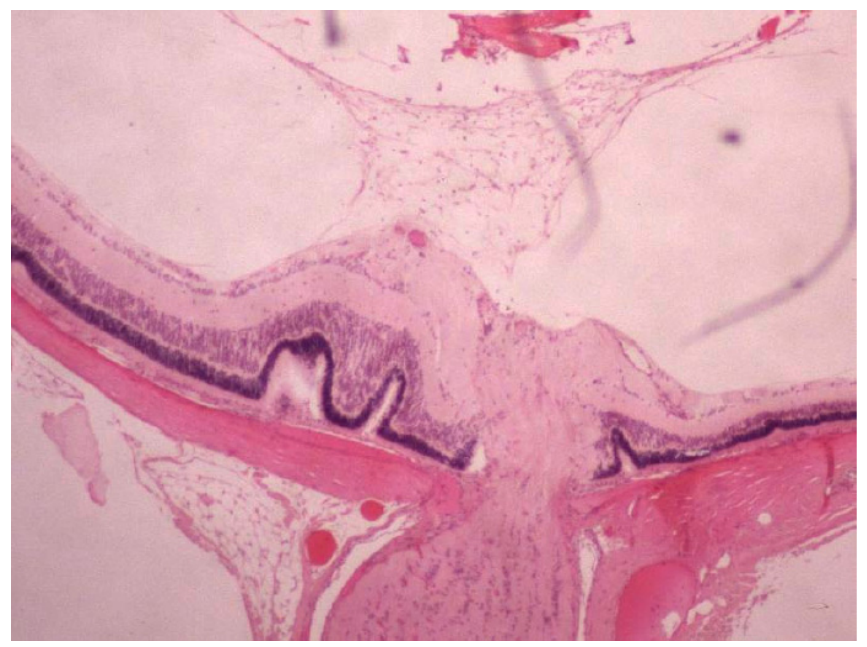

Fig. (6). A tractional retinal detachment around the optic disc in a SDT rat. A tractional retinal detachment with large folds of thickened retina around the optic disc is observed in a 52-week-old male SDT rat (hematoxylin-eosin stain, $4 \mathrm{x}$ ).

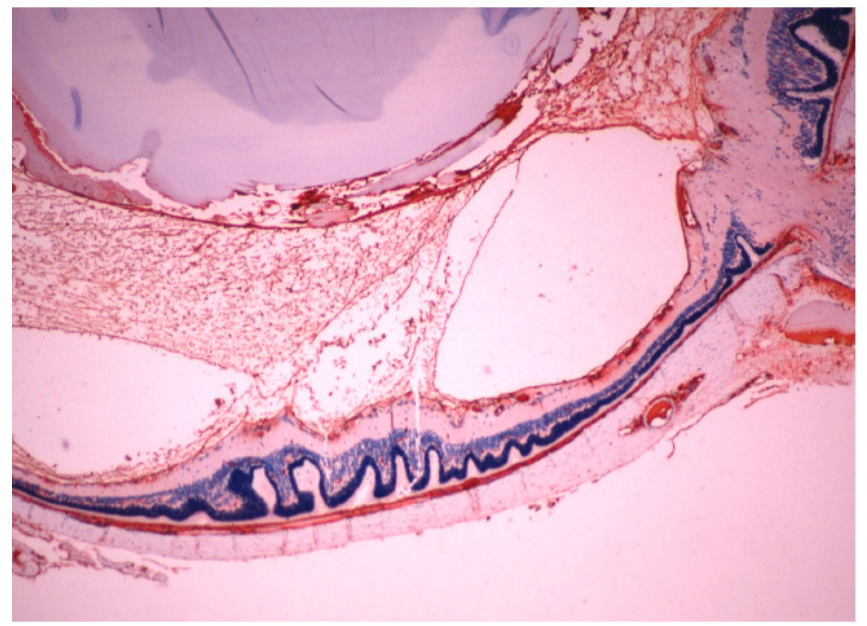

Fig. (7). A tractional retinal detachment in the midperiphery in a SDT rat. A tractional detachment in the midperiphery of the retina is observed in an 82-week-old female SDT rat. Immunoreactivity to albumin is noted around the retinal vessels (immunostaining for albumin, 4x) [6].

Electrophysiologic studies in SDT rats also have been reported $[11,12]$. The a- and b-waves and the oscillatory potentials decreased substantially with prolonged implicit times. These electroretinography findings may reflect vascular and neuronal damage throughout the retina as seen in the advanced stages of human DR.

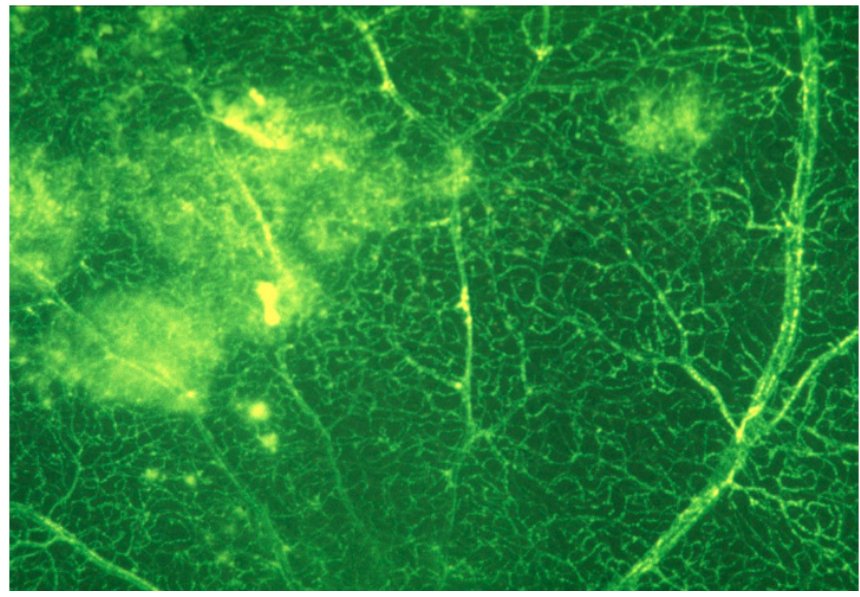

Fig. (8). Fluorescein-dextran angiomicroscopy in the midperiphery in a SDT rat. Fluorescein-dextran angiomicroscopy in a 51-weekold male SDT rat. Extensive hyperfluorescence, suggesting retinal neovascularization, is observed.

\section{NEOVASCULAR GLAUCOMA}

Iris neovascularization occurs frequently in advanced cases of DR in humans. The pathogenesis of iris neovascularization is assumed to be induced by the hypoxic state of the iris itself and VEGF from the hypoxic retina. When the iris neovascularization invades the iris root and anterior chamber angle, the trabecular meshwork becomes dysfunctional and aqueous outflow decreases. These pathological changes cause increased intraocular pressure. In advanced cases, peripheral anterior synechiae develop and completely close the anterior chamber angle; posterior synechiae develop and completely block the aqueous flow from the posterior chamber to the anterior chamber (papillary block); and massive hemorrhages from the fragile iris neovascularization in the anterior chamber occur eventually.

In some advanced cases in SDT rats, iris neovascularization and hemorrhages of the anterior chamber developed (Fig. 9). A neovascular membrane around the pupil may cause posterior synechiae (Fig. 10), and this condition may induce papillary block. Iris neovascularization has not been reported previously in diabetic animals. The SDT rat is the first animal model of diabetic iris neovascularization.

\section{CONCLUSION}

The SDT rat shows three major diabetic ocular complications: cataract, DR, and neovascular glaucoma. An appropriate animal model of diabetic complications is needed to conduct research on the pathogenesis of diabetes and to develop therapy for diabetic complications. Although there are some differences in the diabetic ocular complications between SDT rats and human patients with diabetes, the SDT rat is useful for conducting research into diabetic ocular complications. 


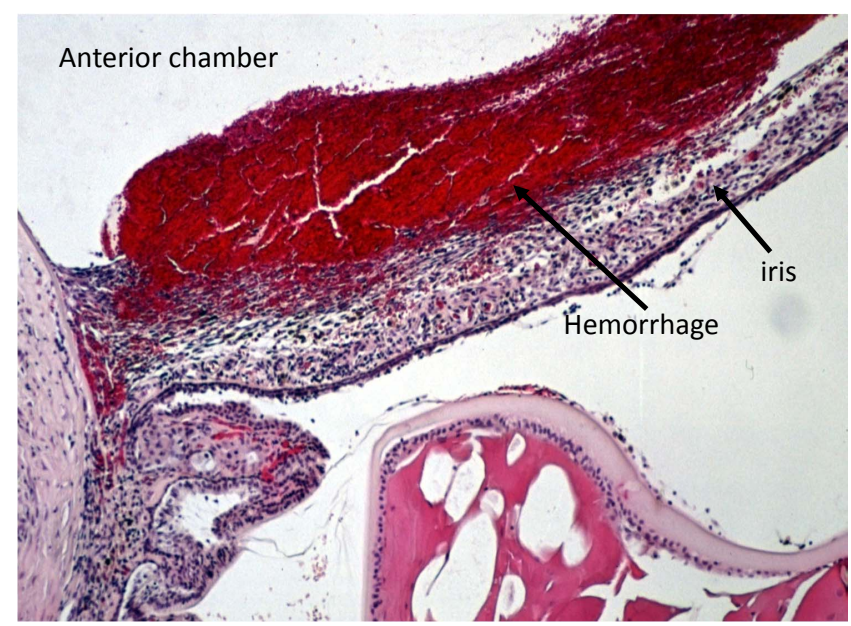

Fig. (9). Hemorrhagic neovascular glaucoma with massive hemorrhage in a SDT rat. A massive hemorrhage in the anterior chamber associated with proliferation around the iris is seen in a 77-week-old male SDT rat (hematoxylin-eosin, 4x) [6].
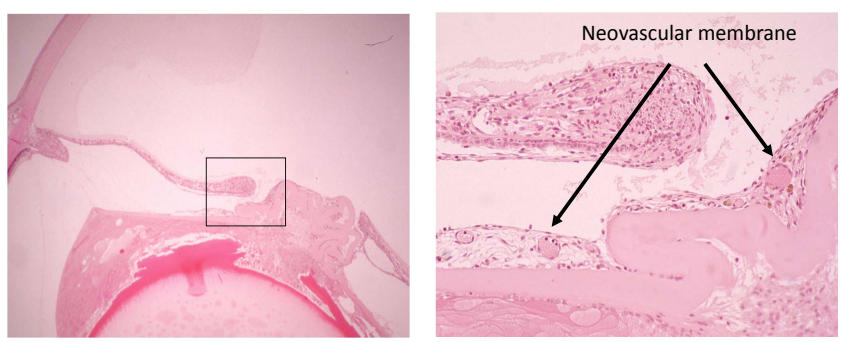

Fig. (10). Posterior synechiae with neovascular membrane in a SDT rat. Posterior synechiae in an 82-week-old female SDT rat. The box indicates the site of the synechiae. A neovascular membrane can be seen around the pupil (hematoxylin-eosin stain, 4x) [6].

\section{REFERENCES}

[1] Resnikoff S, Pascolini D, Etya'ale D, et al. Global data on visual impairment in the year 2002. Bull World Health Organ 2004; 82: 844-51.

[2] DCCT. The effect of intensive treatment of diabetes on the development and progression of long-term complications in insulin-dependent diabetes mellitus. N Engl J Med 1993; 329: 977 86.

[3] UKPDS. Intensive blood-glucose control with sulphonylureas or insulin compared with conventional treatment and risk of complications in patients with type 2 diabetes (UKPDS 33). Lancet 1998; 352: 837-53.

[4] Engerman RL, Bloodworth JM, Jr. Experimental diabetic retinopathy in dogs. Arch Ophthalmol 1965; 73: 205-10.

[5] Kador PF, Takahashi Y, Wyman M, Ferris F, 3rd. Diabeteslike proliferative retinal changes in galactose-fed dogs. Arch Ophthalmol 1995; 113: 352-4.

[6] Kakehashi A, Saito Y, Mori K, et al. Characteristics of diabetic retinopathy in SDT rats. Diabetes Metab Res Rev 2006; 22: 45561.

[7] Shinohara M, Masuyama T, Shoda T, et al. A new spontaneously diabetic non-obese Torii rat strain with severe ocular complications. Int J Exp Diabetes Res 2000; 1: 89-100.

[8] Shinohara M, Masuyama T, Kakehashi A. The spontaneously diabetic Torii (SDT) rat with retinopathy lesions resembling those of humans. In: Shafrir E, Ed. Animal models of diabetes. Boca Raton (FL): CRC Press 2007; pp. 311-21.

[9] Matsuoka M, Ogata N, Minamino K, Higuchi A, Matsumura M. High levels of pigment epithelium-derived factor in the retina of a rat model of type 2 diabetes. Exp Eye Res 2006; 82: 172-8.

[10] Matsuoka M, Ogata N, Minamino K, Matsumura M. Leukostasis and pigment epithelium-derived factor in rat models of diabetic retinopathy. Mol Vis 2007; 13: 1058-65.

[11] Okuno T, Oku H, Sugiyama T, Ikeda T. Electroretinographic study of spontaneously diabetic Torii rats. Doc Ophthalmol 2008; 117 : 191-6.

[12] Sasase T, Morinaga H, Abe T, et al. Protein kinase C beta inhibitor prevents diabetic peripheral neuropathy, but not histopathological abnormalities of retina in spontaneously diabetic Torii rat. Diabetes Obes Metab 2009; 11: 1084-7.

(C) Akihiro Kakehashi; Licensee Bentham Open.

This is an open access article licensed under the terms of the Creative Commons Attribution Non-Commercial License (http://creativecommons.org/licenses/by-nc/3.0/) which permits unrestricted, non-commercial use, distribution and reproduction in any medium, provided the work is properly cited. 